ECCOMAS

\section{Proceedia}

COMPDYN 2021

$8^{\text {th }}$ ECCOMAS Thematic Conference on Computational Methods in Structural Dynamics and Earthquake Engineering

M. Papadrakakis, M. Fragiadakis (eds.) Streamed from Athens, Greece, 28 - 30 June 2021

\title{
STUDY OF BIAXIAL SHEAR FAILURE ENVELOPE OF REINFORCED CONCRETE COLUMNS
}

\author{
Beatrice Belletti ${ }^{1}$, Simone Ravasini ${ }^{1}$ and Andrea Alberici ${ }^{1}$ \\ ${ }^{1}$ Department of Engineering and Architecture \\ University of Parma, Italy \\ Parco Area delle Scienze 181/A \\ beatrice.belletti@unipr.it, andrea.alberici1@studenti.unipr.it,simone.ravasini@unipr.it
}

\begin{abstract}
Several scenarios can trigger bi-axial shear in reinforced concrete (RC) vertical members such as wind actions, earthquake, column loss induced by explosions and/or impacts. The majority of standard codes impose uniaxial shear verification of vertical members and neglect the interaction between shear forces and bending moments acting along the two principal directions of inertia of the transversal cross section. Moreover, bi-axial shear interaction curves proposed by codes are based on the fitting of experimental data conducted on heavily reinforced specimens. In this paper, the bi-axial shear failure envelope of existing $R C$ columns, is investigated. A database previously published by Authors on experimental data on beams and columns tested under bi-axial loading is updated. Afterwards, the experimental shear resistances of the database's members subjected to both monotonic and cyclic loading are compared with analytical predictions. Three different formulations are adopted for the analytical calculation of the uniaxial shear resistance: (i) Model Code 2010, (ii) Eurocode 8 and (iii) the cyclic shear resistance recently proposed by Biskinis and Fardis. Such resistances are adopted to create the bi-axial shear failure envelope. Finally, the shape of the bi-axial shear failure envelope is critically analysed in order to select the formulation that provides the best fitting between experimental and analytical bi-axial shear resistances.
\end{abstract}

Keywords: Reinforced Concrete columns, Bi-axial shear, Analytical prediction.

ISSN:2623-3347 (C) 2021 The Authors. Published by Eccomas Proceedia. Peer-review under responsibility of the organizing committee of COMPDYN 2021. doi: $10.7712 / 120121.8542 .18650$ 


\section{INTRODUCTION}

Seismic as well as wind actions can trigger bi-axial shear in reinforced column members [1]. Moreover, in the robustness assessment of structures, the bi-axial shear demand in beams and columns - associated to vertical element loss scenarios - was also highlighted, even if international codes and guidelines such as Unified Facilities Criteria [2] and Eurocodes [3], [4], are considering only the verifications in the case of uniaxial shear scenarios. Hence, even if the bi-axial shear demand covers almost all the fields of structural engineering, the bi-axial shear resistance is almost neglected in European Codes. Several experimental campaigns were carried out during years to investigate the bi-axial monotonic or cyclic shear behaviour of reinforced concrete columns. Usually, square or rectangular sections were investigated. An early study was conducted by Maruyama et al. [5] in 1979. They observed that the applied lateral loads did not significantly affect the column shear strength until the maximum lateral drift was greater than that corresponding to the maximum monotonic shear strength. Woodward et al. [6] in 1980 conducted several experimental tests on squat reinforced concrete columns with the aim to investigate the effects of both longitudinal and transversal reinforcement ratios. They did not observe a significant increase of the lateral strength with reducing the stirrups spacing. Umehara et al. [7] in 1982 investigated 20 columns with square and rectangular sections subject to unilateral and diagonal loadings. They observed that the shear capacity of squat columns under diagonal loadings could be evaluated based on the response in the two principal directions using interaction domains. In particular, for square section a circumference function can be used to represent the bi-axial shear domain, while an elliptical curve can be used for rectangular sections. In the experimental program by Joh and Shibata [8] in 1984, columns square and rectangular cross-sections were tested under lateral bi-axial loading until achievement of shear failure, using a normalized interaction domain respect to the shear strength along principal directions. Mizoguchi et al. [9] in 1990 investigated the effects of varying axial load on bi-axial loading response of squat columns, observing that columns diagonally loaded had a 5-6\% lower resistance compared with counterparts loaded along principal directions. In more recent studies by Pham et al. [10] and Dang et al. [11] the shear strength mechanisms were associated to the lack of transversal reinforcement and vulnerability of low-rise structures characterized by in-plane and elevation irregularities. The mentioned experimental tests were collected from the authors in a Database [12] and used for comparisons with analytical provisions [13].

Actually, the majority of international normative and guidelines such as Model Code 2010 [14] and Eurocode 8 [15] neglect the interaction between shear and bending acting along the two principal directions of cross section. Conversely, the Japan Society of Civil Engineers (JSCE) guidelines [16] can consider bi-axial loading capacity using an interaction domain.

In this paper, three analytical formulations are considered in order to calculate the uniaxial shear resistance; in particular the formulation proposed by Eurocode 8 [15], Model code 2010 [14] and Biskinis and Fardis [17] .

Afterwards, according to Japanese Code [16], the bi-axial failure criterion is evaluated Finally, the bi-axial shear resistances are compared with the experimental results collected by Authors in their database.

\section{EXPERIMENTAL DATABASE}

In the following, the experimental database collected by Authors is reported in terms of member geometry, reinforcement ratios, mechanical properties and loading conditions. Columns have square or rectangular cross section. The boundary conditions are mostly fixed at the base and allow the lateral displacement at the top according to the so called "shear type" scheme, 
see Figure 1a. Only in the experimental campaign conducted by Dang et al. [11], the boundary conditions allow lateral displacement and rotation at the top of the column. The loading is applied at the top of columns by applying monotonic or cyclic forces, Figure $1 b$.

The axial load can be varied to study its influence of shear resistance and lateral drift capacity.

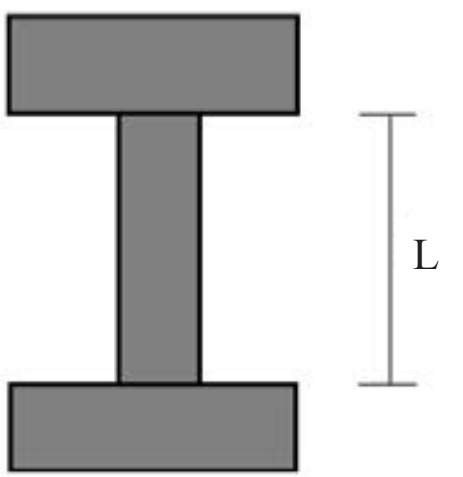

(a)

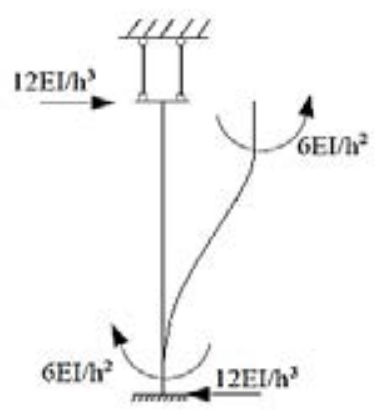

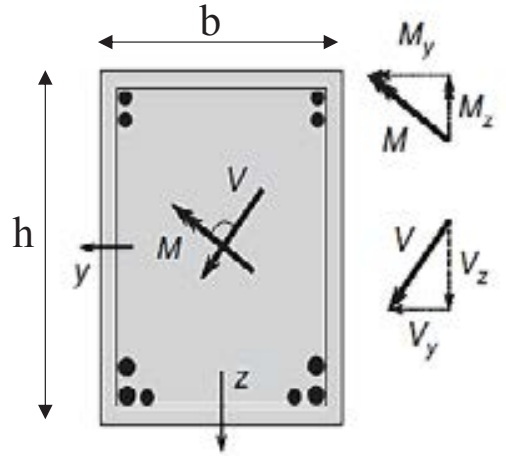

(b)

Figure 1 Test setup: (a) specimen geometry and static scheme; (b) cross-section shear/bending decomposition.

\begin{tabular}{|c|c|c|c|c|c|c|c|c|c|c|c|c|c|c|c|}
\hline $\begin{array}{c}\text { Maruyama et } \\
\text { al. (1979) }\end{array}$ & $\begin{array}{r}\text { Sec } \\
\text { prop }\end{array}$ & $\begin{array}{l}\text { tion } \\
\text { erties }\end{array}$ & & adin & con & dition & & $\begin{array}{l}\text { Long } \\
\text { reinfo }\end{array}$ & $\begin{array}{l}\text { itudinal } \\
\text { rcemen }\end{array}$ & & & & rrups & & $\begin{array}{l}\text { Concrete } \\
\text { strength }\end{array}$ \\
\hline Snecimen & b & $\mathbf{h}$ & $v$ & $\alpha$ & a/d & Loading & d & $\Phi$ & $\rho$ & $\mathbf{f}_{\mathrm{sy}}$ & $\Phi_{w}$ & $\mathbf{s}_{\mathrm{w}}$ & $\rho_{\mathrm{w}}$ & $\mathbf{f}_{\mathrm{yw}}$ & $\mathbf{f}_{\mathrm{c}}$ \\
\hline Specimen & [mm] & {$[\mathrm{mm}]$} & & {$\left[{ }^{\circ}\right]$} & {$[-]$} & History & {$[\mathrm{mm}]$} & {$[\mathrm{mm}]$} & {$[-]$} & {$[\mathrm{MPa}]$} & {$[\mathrm{mm}]$} & {$[\mathrm{mm}]$} & {$[-]$} & [MPa] & {$[\mathrm{MPa}]$} \\
\hline 00-V-0-I Uni & 305 & 305 & 0 & 0 & 2.1 & Cyclic & 219 & 19 & $2.44 \%$ & 448 & 6 & 65 & $0.29 \%$ & 469 & 35 \\
\hline 00-V-V-S-Diag & 305 & 305 & 0 & 45 & - & Cyclic & - & 19 & $2.44 \%$ & 448 & 6 & 65 & $0.29 \%$ & 469 & 35 \\
\hline
\end{tabular}

Table 1 Experimental setup and specimen features, by Maruyama et al. [5].

\begin{tabular}{|c|c|c|c|c|c|c|c|c|c|c|c|c|c|c|c|}
\hline $\begin{array}{l}\text { Woodward et } \\
\text { al. (1980) }\end{array}$ & \multicolumn{2}{|c|}{$\begin{array}{c}\text { Section } \\
\text { properties }\end{array}$} & \multicolumn{4}{|c|}{ Loading condition } & \multicolumn{4}{|c|}{$\begin{array}{l}\text { Longitudinal } \\
\text { reinforcement }\end{array}$} & \multicolumn{4}{|c|}{ Stirrups } & \multirow{2}{*}{\begin{tabular}{|c|} 
Concrete \\
strength
\end{tabular}} \\
\hline \multirow{2}{*}{ Specimen } & b & $\mathbf{h}$ & $v$ & $\alpha$ & a/d & \multirow{2}{*}{$\begin{array}{l}\text { Loading } \\
\text { History }\end{array}$} & d & $\Phi$ & $\rho$ & $\mathbf{f}_{\mathrm{sy}}$ & $\Phi_{w}$ & $\mathbf{s}_{\mathbf{w}}$ & $\rho_{\mathrm{w}}$ & $\mathbf{f}_{\mathrm{yw}}$ & \\
\hline & {$[\mathrm{mm}]$} & {$[\mathrm{mm}]$} & & {$\left[{ }^{\circ}\right]$} & {$[-]$} & & {$[\mathrm{mm}]$} & {$[\mathrm{mm}]$} & {$[-]$} & {$[\mathrm{MPa}]$} & {$[\mathrm{mm}]$} & {$[\mathrm{mm}]$} & {$[-]$} & {$[\mathrm{MPa}]$} & {$[\mathrm{MPa}]$} \\
\hline 0-86-14-DM & 305 & 305 & 0 & 45 & - & Cyclic & - & 19 & $2.44 \%$ & 503 & 6 & 65 & $0.29 \%$ & 503 & 41 \\
\hline C-86-14-DM & 305 & 305 & 0.16 & 45 & - & Cyclic & - & 19 & $2.44 \%$ & 503 & 6 & 65 & $0.29 \%$ & 503 & 36 \\
\hline
\end{tabular}

Table 2 Experimental setup and specimen features, by Woodward et al. [6].

\begin{tabular}{|c|c|c|c|c|c|c|c|c|c|c|c|c|c|c|c|}
\hline \multirow{3}{*}{\begin{tabular}{|c}
$\begin{array}{c}\text { Umehara H. et } \\
\text { al. (1982) }\end{array}$ \\
Specimen
\end{tabular}} & \multicolumn{2}{|c|}{$\begin{array}{c}\text { Section } \\
\text { properties }\end{array}$} & \multicolumn{4}{|c|}{ Loading condition } & \multicolumn{4}{|c|}{$\begin{array}{l}\text { Longitudinal } \\
\text { reinforcement }\end{array}$} & \multicolumn{4}{|c|}{ Stirrups } & \multirow{2}{*}{\begin{tabular}{|c|}
$\begin{array}{c}\text { Concrete } \\
\text { strength }\end{array}$ \\
$\mathbf{f}_{\mathrm{c}}$ \\
\end{tabular}} \\
\hline & b & $\mathbf{h}$ & $v$ & $\alpha$ & a/d & Loading & d & $\Phi$ & $\rho$ & $\mathbf{f}_{\mathrm{sy}}$ & $\Phi_{w}$ & $\mathbf{s}_{\mathrm{w}}$ & $\rho_{\mathrm{w}}$ & $\mathbf{f}_{\mathrm{yw}}$ & \\
\hline & {$[\mathrm{mm}]$} & {$[\mathrm{mm}]$} & & {$\left[{ }^{\circ}\right]$} & {$[-]$} & History & {$[\mathrm{mm}]$} & {$[\mathrm{mm}]$} & {$[-]$} & {$[\mathrm{MPa}]$} & {$[\mathrm{mm}]$} & {$[\mathrm{mm}]$} & {$[-]$} & {$[\mathrm{MPa}]$} & [MPa] \\
\hline OUS & 230 & 410 & 0 & 0 & 1.6 & Cyclic & 284 & 19 & $3.01 \%$ & 441 & 6 & 89 & $0.28 \%$ & 413 & 40 \\
\hline OUW & 410 & 230 & 0 & 0 & 2.4 & Cyclic & 188 & 19 & $3.01 \%$ & 441 & 6 & 89 & $0.31 \%$ & 413 & 40 \\
\hline CUS & 230 & 410 & 0.162 & 0 & 1.6 & Cyclic & 284 & 19 & $3.01 \%$ & 441 & 6 & 89 & $0.28 \%$ & 413 & 35 \\
\hline CUW & 410 & 230 & 0.162 & 0 & 2.4 & Cyclic & 188 & 19 & $3.01 \%$ & 441 & 6 & 89 & $0.31 \%$ & 413 & 35 \\
\hline $2 C U S$ & 230 & 410 & 0.270 & 0 & 1.6 & Cyclic & 284 & 19 & $3.01 \%$ & 441 & 6 & 89 & $0.28 \%$ & 413 & 42 \\
\hline CDS30 & 230 & 410 & 0.132 & 30 & - & Cyclic & - & 19 & $3.01 \%$ & 441 & 6 & 89 & $0.28 \%$ & 413 & 43 \\
\hline CDW30 & 230 & 410 & 0.135 & 60 & - & Cyclic & - & 19 & $3.01 \%$ & 441 & 6 & 89 & $0.55 \%$ & 413 & 42 \\
\hline
\end{tabular}

Table 3 Experimental setup and specimen features, by Humehara et al. [7]. 
Belletti B., Ravasini S., Alberici A.

\begin{tabular}{|c|c|c|c|c|c|c|c|c|c|c|c|c|c|c|c|}
\hline \multirow{3}{*}{ 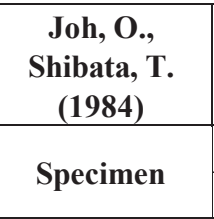 } & \multicolumn{2}{|c|}{$\begin{array}{c}\text { Section } \\
\text { properties }\end{array}$} & \multicolumn{4}{|c|}{ Loading condition } & \multicolumn{4}{|c|}{$\begin{array}{l}\text { Longitudinal } \\
\text { reinforcement }\end{array}$} & \multicolumn{4}{|c|}{ Stirrups } & \multirow{2}{*}{\begin{tabular}{|c}
$\begin{array}{c}\text { Concrete } \\
\text { strength }\end{array}$ \\
$\mathbf{f}_{\mathrm{c}}$ \\
\end{tabular}} \\
\hline & b & $\mathbf{h}$ & $v$ & $\alpha$ & a/d & Loading & d & $\Phi$ & $\rho$ & $\mathbf{f}_{\mathrm{sy}}$ & $\Phi_{w}$ & $\mathbf{s}_{\mathrm{w}}$ & $\rho_{\mathrm{w}}$ & $\mathbf{f}_{\mathrm{yw}}$ & \\
\hline & {$[\mathrm{mm}]$} & {$[\mathrm{mm}]$} & & {$\left[{ }^{\circ}\right]$} & {$[-]$} & & {$[\mathrm{mm}]$} & {$[\mathrm{mm}]$} & {$[-]$} & {$[\mathrm{MPa}]$} & {$[\mathrm{mm}]$} & {$[\mathrm{mm}]$} & {$[-]$} & {$[\mathrm{MPa}]$} & {$[\mathrm{MPa}]$} \\
\hline SS-0-N0 (+) & 300 & & 0 & 0 & 1.7 & & 265 & & $2.25 \%$ & 390.3 & 6 & 63 & $0.30 \%$ & 306.9 & 22.95 \\
\hline SS-0-N0 (-) & 300 & 30 & 0 & 0 & 1.7 & clic & 265 & .1 & $2.25 \%$ & 390.3 & 6 & 63 & $0.30 \%$ & 306.9 & 2.95 \\
\hline SS-0-N1 (+) & 300 & 300 & 0.17 & 0 & 1.7 & & 265 & 19.1 & $2.25 \%$ & 390.3 & 6 & 63 & $0.30 \%$ & 306.9 & 3.34 \\
\hline SS-0-N1 (-) & 300 & 300 & 0.17 & 0 & 1.7 & & 265 & 19.1 & $2.25 \%$ & 390.3 & 6 & 63 & $0.30 \%$ & 306.9 & 3.34 \\
\hline SS-0-N2 (+) & 00 & 3 & 33 & 0 & 1.7 & & 265 & 19.1 & $2.25 \%$ & 390.3 & 6 & 63 & $0.30 \%$ & 306.9 & .24 \\
\hline SS-0-N2 (-) & 00 & 00 & 0.33 & 0 & 1.7 & $\mathrm{Cy}$ & 265 & 19.1 & $2.25 \%$ & 390.3 & 6 & 63 & $0.30 \%$ & 306.9 & 3.24 \\
\hline SS-22.5-NO (+) & 300 & 30 & 0 & 23 & - & & - & 19.1 & $2.25 \%$ & 390.3 & 6 & 63 & $0.30 \%$ & 306.9 & 2.36 \\
\hline SS-22.5-N0 (-) & 300 & 30 & 0 & 23 & - & & - & 19.1 & $2.25 \%$ & 390.3 & 6 & 63 & $0.30 \%$ & 306.9 & 2.36 \\
\hline SS-22.5-N1 (+) & 300 & 20 & 0.17 & 23 & - & & - & 19.1 & $2.25 \%$ & 390.3 & 6 & 63 & $0.30 \%$ & 306.9 & .87 \\
\hline SS-22.5-N1 (-) & 300 & 300 & & 23 & - & & - & 19.1 & $2.25 \%$ & 390.3 & 6 & 63 & $0.30 \%$ & 306.9 & 1.87 \\
\hline $2(+)$ & 300 & 30 & & 23 & - & & - & 19.1 & $2.25 \%$ & 90.3 & 6 & 63 & $0.30 \%$ & 306.9 & 4.71 \\
\hline 5-N2 (-) & 300 & & & 23 & - & & - & & $5 \%$ & 90.3 & 6 & 63 & $.30 \%$ & 6.9 & 4.71 \\
\hline SS-45-N0 (+) & 300 & & 0 & 45 & - & & - & & & & 6 & 63 & $30 \%$ & & \\
\hline SS-45-N0 (-) & & & 0 & 45 & - & & - & & & & 6 & 63 & & & \\
\hline SS-45-N1 (+) & & & & 45 & - & & - & & & & 6 & & & & \\
\hline SS-45-N1 (-) & & & & 45 & - & & - & & $2.25 \%$ & 390.3 & 6 & 63 & $0.30 \%$ & & 3.73 \\
\hline SS-45-N2 (+) & 300 & & & 45 & - & & - & & $2.25 \%$ & 390.3 & 6 & 63 & $0.30 \%$ & & \\
\hline SS-45-N2 (-) & 300 & & & 45 & - & & - & & $2.25 \%$ & 390.3 & 6 & 63 & $0.30 \%$ & & \\
\hline SR-0-N1 (+) & 225 & & 0 & 0 & 1.2 & & 365 & 19.1 & $2.25 \%$ & 390.3 & 5.85 & 71 & $0.34 \%$ & 306.9 & 23.63 \\
\hline SR-0-N1 (-) & 225 & 400 & 0 & 0 & 1.2 & & 365 & 19.1 & $2.25 \%$ & 390.3 & 5.85 & 71 & $0.34 \%$ & 306.9 & 23.63 \\
\hline SR-30-N1 (+) & 225 & 400 & 0.17 & 30 & - & Cyclic & - & 19.1 & $2.25 \%$ & 390.3 & 5.85 & 71 & $0.34 \%$ & 306.9 & 25.10 \\
\hline SR-30-N1 (-) & 225 & 400 & 0.17 & 30 & - & Cyclic & - & 19.1 & $2.25 \%$ & 390.3 & 5.85 & 71 & $0.34 \%$ & 306.9 & 25.10 \\
\hline SR-60-N1 (+) & 225 & 400 & 0.17 & 60 & - & Cyclic & - & 19.1 & $2.25 \%$ & 390.3 & 5.85 & 71 & $0.50 \%$ & 306.9 & 26.28 \\
\hline SR-60-N1 (-) & 225 & 400 & 0.17 & 60 & - & Cyclic & - & 19.1 & $2.25 \%$ & 390.3 & 5.85 & 71 & $0.50 \%$ & 306.9 & 26.28 \\
\hline SR-90-N1 (+) & 400 & 225 & 0.17 & 90 & 2.4 & Cyclic & 190 & 19.1 & $2.25 \%$ & 390.3 & 5.85 & 71 & $0.28 \%$ & 306.9 & 24.81 \\
\hline SR-90-N1 (-) & 400 & 225 & 0.17 & 90 & 2.4 & Cyclic & 190 & 19.1 & $2.25 \%$ & 390.3 & 5.85 & 71 & $0.28 \%$ & 306.9 & 24.81 \\
\hline
\end{tabular}

Table 4 Experimental setup and specimen features, by Joh \& Shibata [8].

\begin{tabular}{|c|c|c|c|c|c|c|c|c|c|c|c|c|c|c|c|}
\hline \multirow{3}{*}{$\begin{array}{c}\text { Pham et al. } \\
(2013)\end{array}$} & \multicolumn{2}{|c|}{$\begin{array}{c}\text { Section } \\
\text { properties }\end{array}$} & \multicolumn{4}{|c|}{ Loading condition } & \multicolumn{4}{|c|}{$\begin{array}{l}\text { Longitudinal } \\
\text { reinforcement }\end{array}$} & \multicolumn{4}{|c|}{ Stirrups } & \multirow{2}{*}{$\begin{array}{c}\begin{array}{r}\text { Concrete } \\
\text { strength }\end{array} \\
\mathbf{f}_{\mathrm{c}}\end{array}$} \\
\hline & $\mathbf{b}$ & $\mathbf{h}$ & $v$ & $\alpha$ & $\mathbf{a} / \mathbf{d}$ & Loading & d & $\Phi$ & $\rho$ & $\mathbf{f}_{\mathrm{sv}}$ & $\Phi_{w}$ & $\mathbf{S}_{\mathbf{w}}$ & $\rho_{\mathrm{w}}$ & $\mathbf{f}_{\mathrm{VW}}$ & \\
\hline & {$[\mathrm{mm}]$} & {$[\mathrm{mm}]$} & & {$\left[{ }^{\circ}\right]$} & {$[-]$} & & {$[\mathrm{mm}]$} & {$[\mathrm{mm}]$} & {$[-]$} & {$[\mathrm{MPa}]$} & {$[\mathrm{mm}]$} & {$[\mathrm{mm}]$} & {$[-]$} & {$[\mathrm{MPa}]$} & {$[\mathrm{MPa}]$} \\
\hline S.C-2.4-0.20 & 350 & 350 & 0.2 & 0 & 3.3 & & 255 & 20 & $2.05 \%$ & 545 & 6 & 125 & $0.13 \%$ & 393 & 23 \\
\hline SC-1.7-0.2 & 350 & 350 & 0.2 & 0 & 2.4 & Cyclic & 255 & 20 & $2.05 \%$ & 545 & 6 & 125 & $0.13 \%$ & 393 & 28 \\
\hline S2.4-30 & 350 & 350 & 0.2 & 30 & - & yclic & - & 20 & $2.05 \%$ & 545 & 6 & 125 & $0.13 \%$ & 511 & 31 \\
\hline S2.4-45 & 350 & 350 & 0.2 & 45 & - & yclic & - & 20 & $2.05 \%$ & 545 & 6 & 125 & $0.13 \%$ & 511 & 29 \\
\hline S1.7-30 & 350 & 350 & 0.2 & 30 & - & & - & 20 & $2.05 \%$ & 545 & 6 & 125 & $0.13 \%$ & 511 & 30 \\
\hline S1.7-45 & 350 & 350 & 0.2 & 45 & - & Cyclic & - & 20 & $2.05 \%$ & 545 & 6 & 125 & $0.13 \%$ & 511 & 29 \\
\hline R.C-1.7-0.35-S & 250 & 490 & 0.35 & 0 & 1.6 & Cyclic & 379 & 20 & $2.05 \%$ & 545 & 6 & 125 & $0.18 \%$ & 393 & 27 \\
\hline R.C-1.7-30 & 250 & 490 & 0.35 & 30 & - & Cyclic & - & 20 & $2.05 \%$ & 545 & 6 & 125 & $0.18 \%$ & 511 & 31 \\
\hline R.C-1.7-45 & 250 & 490 & 0.35 & 45 & - & Cyclic & - & 20 & $2.05 \%$ & 545 & 6 & 125 & $0.18 \%$ & 511 & 30 \\
\hline R.C-1.7-60 & 250 & 490 & 0.35 & 60 & - & Cyclic & - & 20 & $2.05 \%$ & 545 & 6 & 125 & $0.18 \%$ & 511 & 29 \\
\hline
\end{tabular}

Table 5 Experimental setup and specimen features, by Pham et al. [10]. 


\begin{tabular}{|c|c|c|c|c|c|c|c|c|c|c|c|c|c|c|c|}
\hline \multirow{3}{*}{$\begin{array}{c}\begin{array}{c}\text { Mizoguchi et } \\
\text { al.(1990) }\end{array} \\
\text { Specimen }\end{array}$} & \multicolumn{2}{|c|}{$\begin{array}{c}\text { Section } \\
\text { properties }\end{array}$} & \multicolumn{4}{|c|}{ Loading condition } & \multicolumn{4}{|c|}{$\begin{array}{l}\text { Longitudinal } \\
\text { reinforcement }\end{array}$} & \multicolumn{4}{|c|}{ Stirrups } & \multirow{2}{*}{ 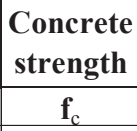 } \\
\hline & $\mathbf{b}$ & h & $v$ & $\alpha$ & a/d & Loading & d & $\Phi$ & $\rho$ & $\mathbf{f}_{\mathrm{sv}}$ & $\Phi_{w}$ & $\mathbf{s}_{\mathrm{w}}$ & $\rho_{\mathrm{w}}$ & $\mathbf{f}_{\mathrm{Vw}}$ & \\
\hline & {$[\mathrm{mm}]$} & {$[\mathrm{mm}]$} & & {$\left[{ }^{\circ}\right]$} & {$[-]$} & History & {$[\mathrm{mm}]$} & {$[\mathrm{mm}]$} & {$[-]$} & {$[\mathrm{MPa}]$} & {$[\mathrm{mm}]$} & {$[\mathrm{mm}]$} & {$[-]$} & {$[\mathrm{MPa}]$} & {$[\mathrm{MPa}]$} \\
\hline 0V04+ & 180 & 180 & 0.44 & 0 & 1.7 & Cyclic & 132 & 12.7 & $3.13 \%$ & 342.3 & 4 & 41 & $0.51 \%$ & 273 & 26 \\
\hline 0V04- & 180 & 180 & 0 & 0 & 1.7 & Cyclic & 132 & 12.7 & $3.13 \%$ & 342.3 & 4 & 41 & $0.51 \%$ & 273 & 26 \\
\hline 0V15+ & 180 & 180 & 0.57 & 0 & 1.7 & Cyclic & 132 & 12.7 & $3.13 \%$ & 342.3 & 4 & 41 & $0.51 \%$ & 273 & 26 \\
\hline 0V15- & 180 & 180 & -0.1 & 0 & 1.7 & Cyclic & 132 & 12.7 & $3.13 \%$ & 342.3 & 4 & 41 & $0.51 \%$ & 273 & 26 \\
\hline 0VA04+ & 180 & 180 & 0.44 & 0 & 1.7 & Cyclic & 132 & 12.7 & $3.13 \%$ & 342.3 & 4 & 41 & $0.51 \%$ & 273 & 27 \\
\hline 0VA04- & 180 & 180 & 0 & 0 & 1.7 & Cyclic & 132 & 12.7 & $3.13 \%$ & 342.3 & 4 & 41 & $0.51 \%$ & 273 & 27 \\
\hline 0VA15+ & 180 & 180 & 0.54 & 0 & 1.7 & Cyclic & 132 & 12.7 & $3.13 \%$ & 342.3 & 4 & 41 & $0.51 \%$ & 273 & 27 \\
\hline 0VA15- & 180 & 180 & -0.1 & 0 & 1.7 & Cyclic & 132 & 12.7 & $3.13 \%$ & 342.3 & 4 & 41 & $0.51 \%$ & 273 & 27 \\
\hline $2 \mathrm{V04+}$ & 180 & 180 & 0.39 & 23 & - & Cyclic & - & 12.7 & $3.13 \%$ & 342.3 & 4 & 41 & $0.51 \%$ & 273 & 31 \\
\hline 2V04- & 180 & 180 & 0 & 23 & - & Cyclic & - & 12.7 & $3.13 \%$ & 342.3 & 4 & 41 & $0.51 \%$ & 273 & 31 \\
\hline 2VA04+ & 180 & 180 & 0.38 & 23 & - & Cyclic & - & 12.7 & $3.13 \%$ & 342.3 & 4 & 41 & $0.51 \%$ & 273 & 31 \\
\hline 2VA04- & 180 & 180 & 0 & 23 & - & Cyclic & - & 12.7 & $3.13 \%$ & 342.3 & 4 & 41 & $0.51 \%$ & 273 & 31 \\
\hline 4V04+ & 180 & 180 & 0.4 & 45 & - & Cyclic & - & 12.7 & $3.13 \%$ & 342.3 & 4 & 41 & $0.51 \%$ & 273 & 29 \\
\hline 4V04- & 180 & 180 & 0 & 45 & - & Cyclic & - & 12.7 & $3.13 \%$ & 342.3 & 4 & 41 & $0.51 \%$ & 273 & 29 \\
\hline $4 \mathrm{~V} 15+$ & 180 & 180 & 0.52 & 45 & - & Cyclic & - & 12.7 & $3.13 \%$ & 342.3 & 4 & 41 & $0.51 \%$ & 273 & 29 \\
\hline 4V15- & 180 & 180 & -0.1 & 45 & - & Cyclic & - & 12.7 & $3.13 \%$ & 342.3 & 4 & 41 & $0.51 \%$ & 273 & 29 \\
\hline 4VA04+ & 180 & 180 & 0.4 & 45 & - & Cyclic & - & 12.7 & $3.13 \%$ & 342.3 & 4 & 41 & $0.51 \%$ & 273 & 29 \\
\hline 4VA04- & 180 & 180 & 0 & 45 & - & Cyclic & - & 12.7 & $3.13 \%$ & 342.3 & 4 & 41 & $0.51 \%$ & 273 & 29 \\
\hline 4VA15+ & 180 & 180 & 0.5 & 45 & - & Cyclic & - & 12.7 & $3.13 \%$ & 342.3 & 4 & 41 & $0.51 \%$ & 273 & 30 \\
\hline 4VA15- & 180 & 180 & -0.1 & 45 & - & Cyclic & - & 12.7 & $3.13 \%$ & 342.3 & 4 & 41 & $0.51 \%$ & 273 & 30 \\
\hline
\end{tabular}

Table 6 Experimental setup and specimen features, by Mizoguchi et al. [9].

\begin{tabular}{|c|c|c|c|c|c|c|c|c|c|c|c|c|c|c|c|}
\hline \multirow{3}{*}{$\begin{array}{c}\text { Dang et al. } \\
(2018)\end{array}$} & \multicolumn{2}{|c|}{$\begin{array}{c}\text { Section } \\
\text { properties }\end{array}$} & \multicolumn{4}{|c|}{ Loading condition } & \multicolumn{4}{|c|}{$\begin{array}{l}\text { Longitudinal } \\
\text { reinforcement }\end{array}$} & \multicolumn{4}{|c|}{ Stirrups } & \multirow{2}{*}{\begin{tabular}{|c} 
Concrete \\
strength
\end{tabular}} \\
\hline & $\mathbf{b}$ & $\mathbf{h}$ & $v$ & $\alpha$ & a/d & Loading & d & $\Phi$ & $\rho$ & $\mathbf{f}_{\mathrm{sy}}$ & $\boldsymbol{\Phi}_{\mathrm{w}}$ & $\mathbf{s}_{\mathbf{w}}$ & $\rho_{\mathrm{w}}$ & $\mathbf{f}_{\mathrm{yw}}$ & \\
\hline & {$[\mathrm{mm}]$} & {$[\mathrm{mm}]$} & & {$\left[{ }^{\circ}\right]$} & {$[-]$} & History & {$[\mathrm{mm}]$} & {$[\mathrm{mm}]$} & {$[-]$} & {$[\mathrm{MPa}]$} & {$[\mathrm{mm}]$} & {$[\mathrm{mm}]$} & {$[-]$} & {$[\mathrm{MPa}]$} & {$[\mathrm{MPa}]$} \\
\hline FS 10 U (+) & 300 & 300 & 0.1 & 0 & 3 & Cyclic & 254 & 19 & $2.52 \%$ & 400 & 10 & 200 & $0.26 \%$ & 400 & 27 \\
\hline FS 10 U (-) & 300 & 300 & 0.1 & 0 & 3 & Cyclic & 254 & 19 & $2.52 \%$ & 400 & 10 & 200 & $0.26 \%$ & 400 & 27 \\
\hline FS 10 B (+) & 300 & 300 & 0.1 & 17 & - & Cyclic & - & 19 & $2.52 \%$ & 400 & 10 & 200 & $0.26 \%$ & 400 & 27 \\
\hline FS 10 B (-) & 300 & 300 & 0.1 & 17 & - & Cyclic & - & 19 & $2.52 \%$ & 400 & 10 & 200 & $0.26 \%$ & 400 & 27 \\
\hline SC $10 \mathrm{U}(+)$ & 300 & 300 & 0.1 & 0 & 3 & Cyclic & 254 & 19 & $2.52 \%$ & 400 & 10 & 300 & $0.17 \%$ & 400 & 27 \\
\hline SC 10 U (-) & 300 & 300 & 0.1 & 0 & 3 & Cyclic & 254 & 19 & $2.52 \%$ & 400 & 10 & 300 & $0.17 \%$ & 400 & 27 \\
\hline SC 10 B (+) & 300 & 300 & 0.1 & 17 & - & Cyclic & - & 19 & $2.52 \%$ & 400 & 10 & 300 & $0.17 \%$ & 400 & 27 \\
\hline SC 10 B (+) & 300 & 300 & 0.1 & 17 & - & Cyclic & - & 19 & $2.52 \%$ & 400 & 10 & 300 & $0.17 \%$ & 400 & 27 \\
\hline
\end{tabular}

Table 7 Experimental setup and specimen features, by Dang et al. [11]. 


\section{ANALYTICAL FORMULATIONS}

In this paragraph, the analytical formulations provided by Eurocode 8 [15], MC 2010 [14] and Biskinis \& Fardis [17] model are briefly reported.

\section{1 $\quad$ Eurocode 8}

Firstly, the formulation provided by Eurocode 8 [15] is reported. The yield chord rotation capacity is evaluated for beams and columns, Eq. (1):

$$
\theta_{y}=\phi_{y} \frac{L_{v}}{3}+0.0013\left(1+1.5 \frac{h}{L_{v}}\right)+0.13 \phi_{y} \frac{d_{b} f_{y}}{\sqrt{f_{c}}}
$$

The ultimate chord rotation capacity is evaluated through, Eq. (2):

$$
\theta_{u}=\frac{1}{\gamma_{e l}} 0.016 \cdot\left(0.3^{v}\right)\left[\frac{\max \left(0.01 ; \omega^{\prime}\right)}{\max (0.01 ; \omega)} f_{c}\right]^{0.225}\left(\frac{L_{v}}{h}\right)^{0.35} 25^{\left(\alpha \rho_{x} \frac{f_{m}}{f_{c}}\right)}\left(1.25^{100 \rho_{d}}\right)
$$

The strength degradation due to cyclic loading is calculated as a function of the ductility $\mu_{\Delta p l}$ which is calculated by the ratio between the plastic and the yield chord rotation, Eq. (3):

$$
\mu_{\Delta p l}=\frac{\theta_{u}}{\theta_{y}}-1
$$

The value of $\mu_{\Delta p l}$ may vary between 0 and 5, corresponding to null and maximum degradation, respectively. The resisting shear strength $V_{r}$ under cyclic conditions can be determined using Eq. (4):

$$
V_{r}=\frac{1}{\gamma_{c l}}\left\{\frac{h-x}{2 L_{v}} \min \left(N ; 0.55 A_{c} f_{c}\right)+\left[1-0.005 \min \left(5 ; \mu_{\Delta p l}\right)\right]\left\{0.16 \max \left[0.5 ; 100 \rho_{\text {tot }}\right]\left[1-0.16 \min \left(5 ; \frac{L_{v}}{h}\right)\right] \sqrt{f_{c}} A_{c}\right\}+V_{w}\right\}
$$

Three contributions are considered: the axial load magnitude $N$, steel reinforcement ratio $\rho_{\text {tot }}$ and the plastic ductility $\mu_{\Delta p l}$.

\subsection{Model Code 2010}

The Model Code 2010 [14] formulation is based on in the Level of Approximation (LoA) framework for the evaluation of the shear resistance. LoA I and II are used for both members with and without transverse reinforcement while LoA III can be used only for members with shear reinforcement. Since stirrups are provided in all the analysed members, in this paper the LoA III is used for the calculation of the shear resistance. The longitudinal strain $\varepsilon_{x}$ is evaluated in correspondence of the mid-depth of the effective shear depth as follows, Eq. (5):

$$
\varepsilon_{x}=\frac{1}{2 \cdot E_{s} \cdot A_{s}}\left[\frac{M_{E d}}{z}+V_{E d}+N_{E d}\left(\frac{1}{2} \mp \frac{\Delta e}{z}\right)\right]
$$

The shear resistance $V_{R d}$ for elements with stirrups is calculated through Eq. (6):

$$
V_{R d}=V_{R d, c}+V_{R d, s} \leq V_{R d, \text { max }}\left(\theta_{\min }\right)
$$

To consider the cyclic degradation effects, Model Code 2010 imposes the use of the following strut inclination angles (linear interpolation is allowed):

- wherever the plastic part of the chord rotation demand is more than twice the elastic part $\theta_{y}$, set $\operatorname{cotg}(\theta)=1$.

- for elastic flexural response, with zero plastic chord rotation, set $\operatorname{cotg}(\theta)=2.5$. 


\subsection{New proposal by Biskinis and Fardis}

The new proposal by Biskinis and Fardis [17] for the assessment of the cyclic shear resistance is based on the monotonic shear resistance of Model Code 2010. The new model allows to consider the degradation effect associated to cyclic loading, by applying the following modifications to the shear resistance formulation proposed in Model Code 2010:

- The longitudinal strain $\varepsilon_{x}$ is firstly evaluated in correspondence of the section middepth:

$$
\varepsilon_{x}=\frac{1}{2 A_{s} E_{s}}\left(\mu \frac{M_{E d}}{z}+\frac{N_{E d}}{2}+V_{E d}\right) \leq \min \left(0.02 ; \frac{1}{2 A_{s} E_{s}}\left(\mu \frac{\max M}{z}+\frac{N}{2}\right)\right)
$$

- The contribution due to the axial load $N$ in columns is considered by adding the term $V_{R, N}$ given in Eq.(8) :

$$
V_{R, N}=N \frac{h-x}{2 L_{s}}
$$

Where $x$ is the neutral axis and $L_{s}$ is the shear span.

- The contribution of transverse steel to shear resistance is:

$$
V_{R, S}=\left(\rho_{w} b_{w} z f_{y w}\right) \cot (\theta)
$$

Where the upper limit on the yield stress of shear reinforcement is set equal to $f_{y w}=\min \left(f_{y w} ; 6 / \rho_{w}\right)$.

- The contribution of concrete to shear resistance is:

$$
\left\{\begin{array}{c}
k_{V}=\frac{0.4}{1+1500 \varepsilon_{x}}\left(1-\frac{V_{E d}}{V_{R d, \max }\left(\theta_{\min }\right)}\right) \\
V_{R d, c}=k_{V} \frac{\sqrt{f_{c k}}}{\gamma_{c}} z \cdot b_{w}
\end{array}\right.
$$

The shear resistance is calculated based on the selected Level of Approximation as follows:

- For LoA II: $V_{R}=V_{R, N}+V_{R, s} \leq V_{R, \max }$

- For LoA III: $V_{R}=V_{R, N}+V_{R, s}+V_{R, c} \leq V_{R, \max }$

In this study, the Level of Approximation III is used. Note average mechanical properties and partial safety factors equal to 1 are used to compare analytical and experimental shear resistances. The comparisons of the shear capacity in terms of resistance and ductility obtained by using the three formulations are reported in Figure 2.

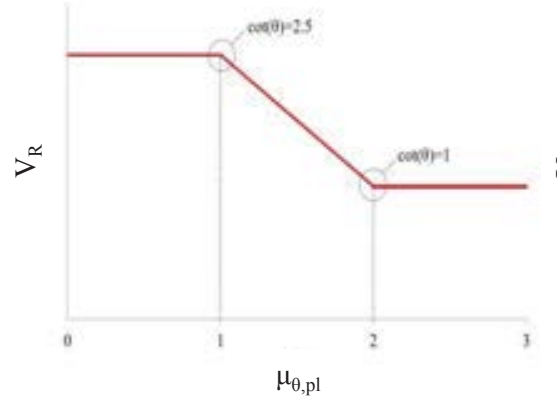

(a)

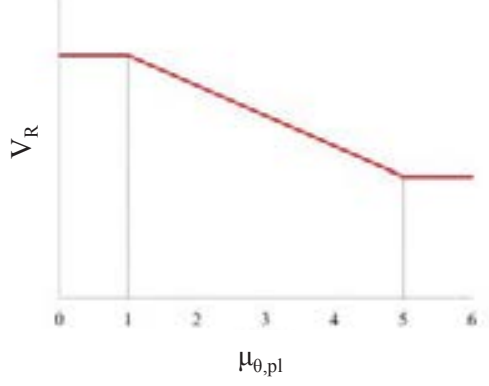

(b)

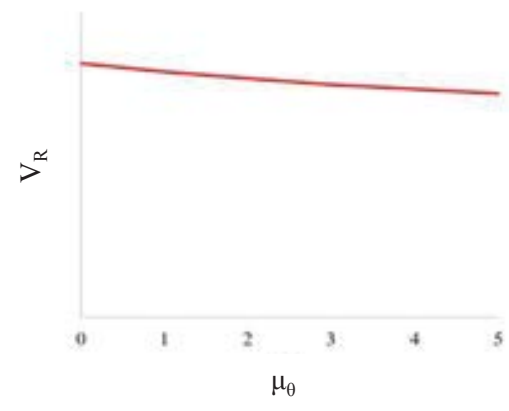

(c)

Figure 2 Capacity curves for: (a) Model Code 2010 [14]; (b) Eurocode 8 [15]; (c) Biskinis and Fardis [17]. 
The previously explained formulations are adopted to calculate the shear resistance under monotonic or cyclic loading along the two principal directions of the transversal cross section of the column. Afterward, to account for bi-axial shear resistance, the interaction domain proposed by JSCE [16] is used, according to Eq. (11):

$$
\left(\gamma_{i} \frac{V_{E d, x}}{V_{R d, x}}\right)^{2}+\left(\gamma_{i} \frac{V_{E d, y}}{V_{R d, y}}\right)^{2}=1
$$

In Figure 3 are reported possible failure modes of RC columns, based on the intersection between bilinear flexural and shear (based on EC8 [15] approach) capacities curves. Indeed, three failure modes are possible based on the intersection between simplified bilinear flexural capacity and shear resistance provided: (i) flexural failure, without intersection with shear capacity, (ii) mixed flexural-shear failure after achievement of flexural yielding, (iii) shear failure before flexural yielding. In this study, shear and mixed flexural-shear failure modes are considered.

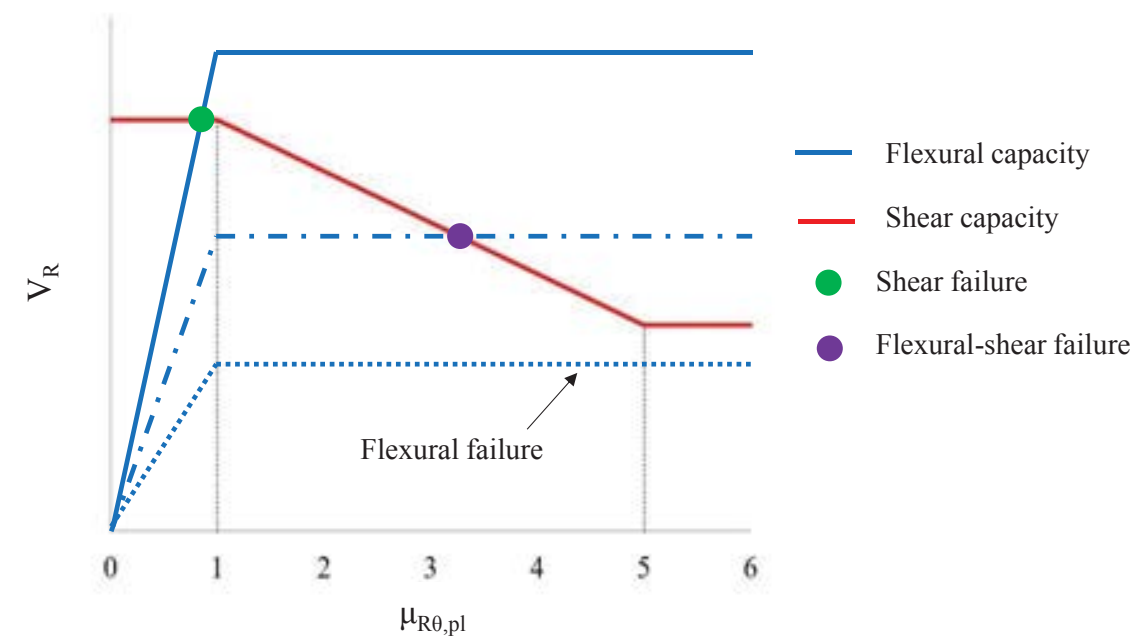

Figure 3 Exemplificative flexural vs shear (EC8 [15] approach) capacities and failure modes.

\section{RESULTS AND DISCUSSION}

In this paragraph the bi-axial domains obtained from experimental results and analytical formulations are plotted and compared. Average mechanical properties and partial safety factors equal to 1 are used for calculations. Figure 4 and Figure 5 are related to specimens without and with compressive axial load, respectively.

Figure 4 shows that the analytical provision by Biskinis \& Fardis [17] tends to underestimate the shear resistance compared to Model Code 2010 and Eurocode 8 provisions. This is attributed to the reduction coefficient equal to 1.8 introduced in the $k_{\varepsilon}$ formulation by Biskinis $\&$ Fardis [17] which accounts for cyclic loading. Indeed, this coefficient affects the magnitude of $V_{R d, \max }$ calculated at $\theta_{\min }$ equal to $21.8^{\circ}$ as provided for LoA III, see Eq. (12):

$$
\left\{\begin{array}{c}
k_{\varepsilon}=\frac{1}{1.8\left(1.2+55 \varepsilon_{1}\right)} \\
V_{R d, \max }\left(\theta_{\min }\right)=k_{\varepsilon} \cdot \eta_{f c} \cdot \frac{f_{c k}}{\gamma_{c}} \cdot b_{w} \cdot z \cdot \sin \left(\theta_{\min }\right) \cdot \cos \left(\theta_{\min }\right)
\end{array}\right.
$$

As consequence, the coefficient $k_{V}$ and the corresponding concrete shear resistance contribution $V_{R d, c}$ reported in Eq. (10) are relatively small. 


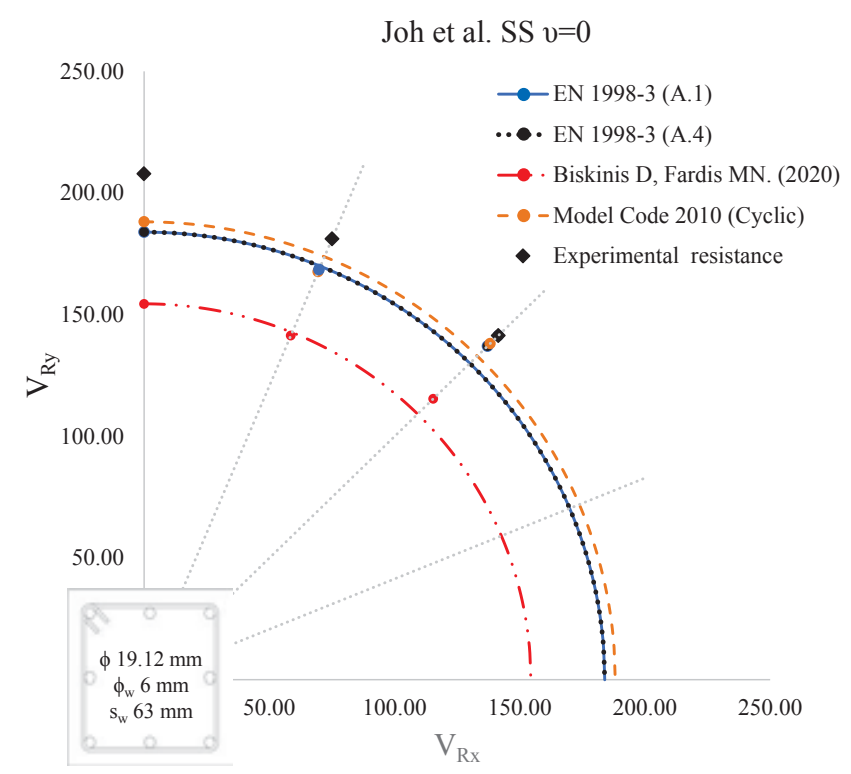

(a)

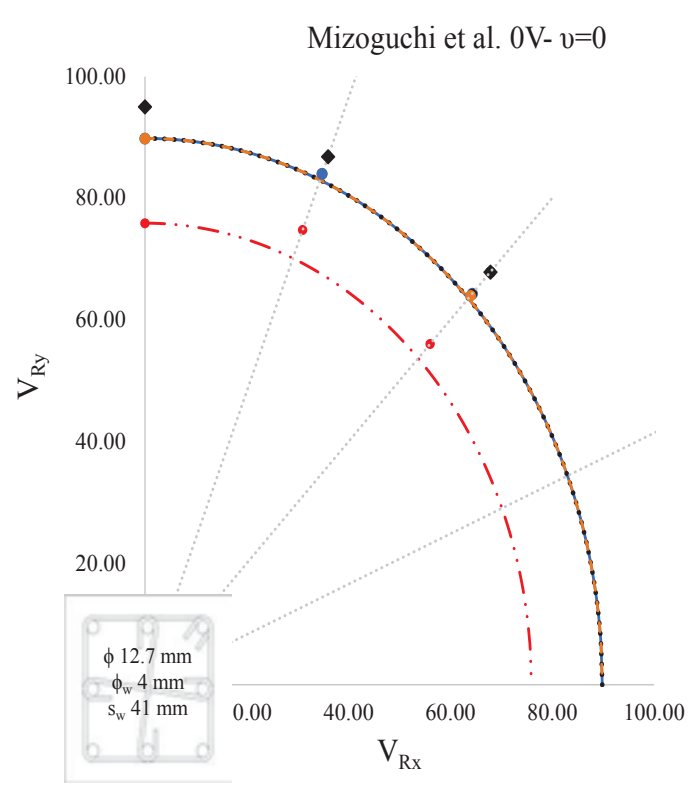

(b)

Figure 4 Interaction domains N = 0: (a) Joh \& Shibata specimen SSN0-; (b) Mizoguchi et al. specimen 0V-.

Figure 5 shows that in the case of compressive axial force applied to columns, the analytical approaches proposed in Eurocode 8 and by Biskinis \& Fardis provide a better fitting of the experimental results. Indeed, the former approaches consider the additional beneficial term related to the compressive axial load contribution, which for Eurocode 8 is defined by Eq. (13), while for Biskinis \& Fardis [17], is defined by Eq. (8):

$$
V_{R, N}=\frac{h-x}{2 L_{s}} \min \left(N ; 0.55 A_{c} f_{c}\right)
$$

Conversely to the mentioned approaches, the Model Code 2010 consider the effect of the axial force only in the strains' calculation of the longitudinal reinforcement, according Eq. (5).

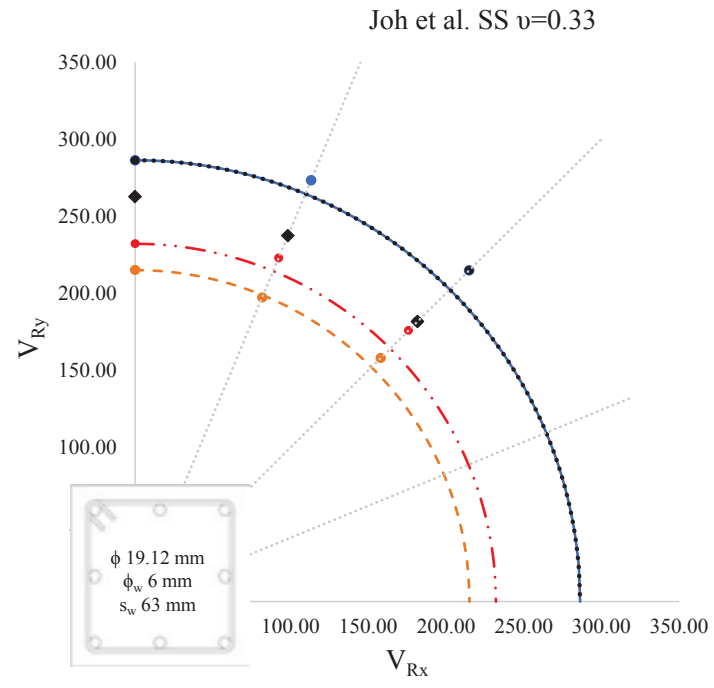

(a)

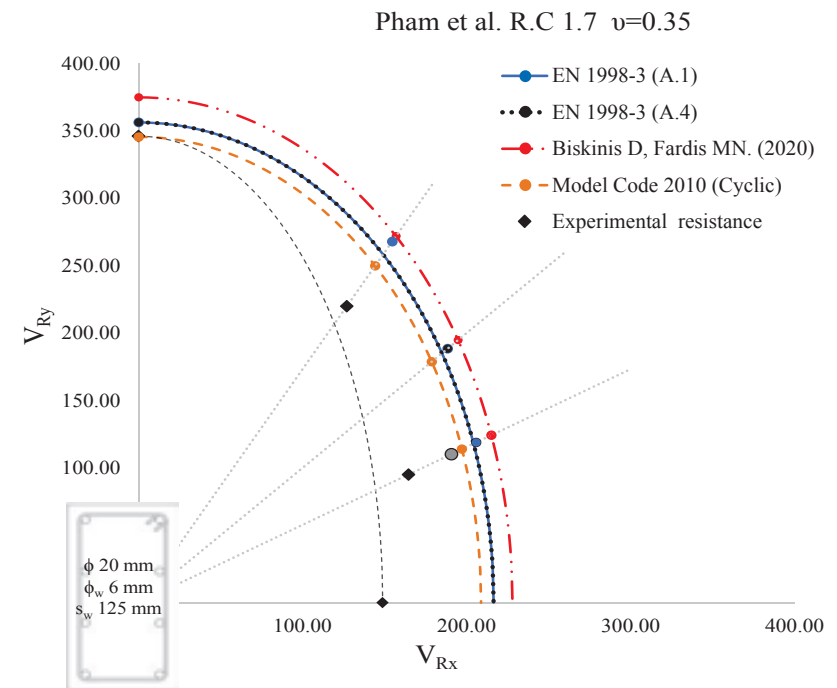

(b)

Figure 5 Interaction domains N < 0: (a) Joh \& Shibata specimen SSN2+; (b) Pham et al. specimen R.C1.7. 
Moreover, by analysing the formulations in Eq. (8) and (13), it is evident the dependency on the cross-section height $h$, the shear span $L_{s}$ and neutral axis depth $x$. This latter term is fundamental and can be calculated using different assumptions based in the cross-section deformability level: (i) at ultimate limit state considering tensile rebars at yield and concrete at a compressive strain of $3.5 \%$, (ii) at the elastic state, where compressive concrete strain is below $2 \%$, thus the hypothesis of linear stresses is valid. Therefore, the assumption adopted to calculate the neutral axis should be coherent with the assumed ductility level.

\section{CONCLUSIONS}

This paper treats the calculation of the bi-axial shear resistance of reinforced concrete columns under cyclic loading. Such shear capacity is calculated by adopting the interaction domains provided by JSCE, starting from the uniaxial resistances of columns calculated along the principal axes of the transversal cross section. Moreover, three different analytical approaches from Eurocode 8, Model Code 2010 and the new proposal by Biskinis \& Fardis are compared with available experimental tests collected in a Database created by Authors. The results show that:

- In absence of axial force, the interaction domains calculated from Model Code 2010 (LoA III) provide higher resistances than Eurocode 8 and Biskinis \& Fardis approaches and a better fitting of experimental evidences. This is attributed to the fact that in the new formulation by Biskinis \& Fardis, a reduction coefficient equal to 1.8 - which accounts for cyclic loading - is applied to the concrete compressive strut resistance. This leads to a considerable reduction of concrete contribution to shear resistance.

- In presence of compressive axial force, the interactions domains calculated from Model Code 2010 (LoA III) provide lower resistances compared to Eurocode 8 and Biskinis \& Fardis approaches. This is attributed to the additional term accounting for the beneficial contribution of compressive axial load. The new proposal from Biskins \& Fardis provide a better fitting of experimental evidences compared to the other analytical provisions considered in this paper.

\section{REFERENCES}

[1] Biskinis, D. \& Fardis, M. N., "Report No. SEE 2009-01 - Deformations of concrete members at yielding and ultimate under monotonic or cyclic loading (including repaired and retrofitted members)," 2009.

[2] DoD, Unified Facilities Criteria (UFC 4-023-03): Design of Buildings To Resist Progressive Collapse. Department of Defense, Washington DC, 2009.

[3] CEN-EC1, EN 1991-1-7: Eurocode 1 - actions on structures: general actions accidental actions. Brussels: European Committee for Standardization, 2006.

[4] CEN-EC2, EN 1992: Eurocode 2: Design of concrete structures - Part 1-1: General rules and rules for buildings. Brussels: European Committee for Standardization, 2004.

[5] Maruyama, K. \& Jirsa, J. O., "CESRL Report No. 79-1 - Shear Behavior of Reinforced Concrete Members under Bidirectional Reversed Lateral Loading," 1979.

[6] Woodward, K. A. \& Jirsa, J. O., "Behavior classification of short Reinforced Concrete columns subject to cyclic deformations," 1980.

[7] Umehara, H. \& Jirsa, J., "PMFSEL Report No. 82-3 - Shear strength and deterioration 
of short reinforced concrete columns under cyclic deformations," 1982.

[8] Joh, O. \& Shibata, T., "Shear failure of reinforced concrete columns due to biaxial lateral forces," in Proceedings of the 8th World Conferance on Earthquake Engineering San Francisco, 1984, pp. 577-584.

[9] Mizoguchi, M., Arakawa, T., Arai, Y. \& Yoshida, M., "Shear resisting behavior of short Reinforced Concrete columns under biaxial bending-shear and varying axial load," Trans. Japan Concr. Inst., vol. 12, pp. 347-354, 1990.

[10] Pham, T. P. \& Li, B., "Seismic behavior of reinforced concrete columns with light transverse reinforcement under different lateral loading directions," ACI Struct. J., vol. 110, no. 5, pp. 833-843, 2013, doi: 10.14359/51685836.

[11] Dang, H. V., Lee, K., Han, S. W. \& Kim, S. J., "Experimental assessment of the effects of biaxial bending moment and axial force on reinforced concrete corner columns," Struct. Concr., vol. 19, no. 4, pp. 1063-1078, 2018, doi: 10.1002/suco.201700211.

[12] Tinini, A., Minelli, F., Belletti, B. \& Scolari, M., "Biaxial shear in RC square beams: Experimental, numerical and analytical program," Eng. Struct., vol. 126, pp. 469-480, 2016, doi: 10.1016/j.engstruct.2016.07.056.

[13] Minelli, F., Tinini, A., Belletti, B. \& Scolari, M., "Studio sperimentale sul taglio biassiale in travi a sezione quadrata," in $20^{\circ}$ Congresso CTE. Milano, Italia; $6-8$ Novembre: $77-87,2014$, p. 8.

[14] Ceb-Fip, fib Model Code for Concrete Structures 2010. 2013.

[15] CEN-EC8, EN 1998: Eurocode 8: Design of structures for earthquake resistance Part 1: General rules, seismic actions and rules for buildings. Brussels: European Committee for Standardization, 2004.

[16] JSCE, Standard Specifications for Concrete Structures. Japan Society of Civil Engineers, 2007.

[17] Biskinis, D. \& Fardis, M. N., "Cyclic shear resistance for seismic design, based on monotonic shear models in fib Model Code 2010 and in the 2018 draft of Eurocode 2," Struct. Concr., vol. 21, no. 1, pp. 129-150, 2020, doi: 10.1002/suco.201900037. 